\title{
On some new Ostrowski type inequalities for co-ordinated s-Godunova-Levin convex functions in the second sense
}

\author{
Seda Kılınç Yıldırım ${ }^{1}$, Hüseyin Budak ${ }^{2}$ and Hüseyin Yıldırım ${ }^{3}$ \\ 1,3 Department of Mathematics, Faculty of Science and Arts, University of Kahramanmaraş Sütçü İmam, Kahramanmaraş, Turkey \\ ${ }^{2}$ Department of Mathematics, Faculty of Science and Arts, University of Düzce,Düzce, Turkey
}

Received: 13 June 2021, Accepted: 16 June 2021

Published online: 10 July 2021.

Abstract: The aim of this paper, some new Ostrowski type inequalities for co-ordinated s-Godunova -Levin convex functions in the second sense are obtained.

Keywords: Co-ordinated $s$-gudunova levin convex function, Hermite-Hadamard inequality,Ostrowski inequality.

\section{Introduction and Preliminaries}

Let $f:[a, b] \rightarrow R$ be continuous on $[a, b]$ and differentiable in $(a, b)$ and assume $\left|f^{\prime}(x)\right| \leq M$ for all $x \in(a, b)$. Then the following holds [1]:

$$
|f(x)-M(f ; a, b)| \leq \frac{M}{b-a} \frac{(b-x)^{2}+(x-a)^{2}}{2}
$$

for all $x \in[a, b]$. Where $M(f ; a, b)=\frac{1}{b-a} \int_{a}^{b} f(x) d x$.

The inequality (1) can be rewritten in equivalent form as;

$$
\left|f(x)-\frac{1}{b-a} \int_{a}^{b} f(t) d t\right| \leq\left[\frac{(x-a)^{2}+(b-x)^{2}}{2(b-a)}\right]\left\|f^{\prime}\right\|_{\infty} .
$$

where $\left\|f^{\prime}\right\|_{\infty}:=\sup _{t \in(a, b)}\left|f^{\prime}(t)\right|<\infty$.

Inequality (1) is well known in the literature as Ostrowski Inequality. Over the years, numerous studies have focused on generalize this inequality. There are numerous generalizations, variants and extensions in the literature, see [4-17] and the references cited therein.

Definition 1. A function $f: \Delta:=[a, b] \times[c, d] \rightarrow \mathbb{R}$ is called co-ordinated convex on $\Delta$, for all $(\kappa, u),(\gamma, v) \in \Delta$ and $\tau, s \in[0,1]$, if it satisfies the following inequality:

$$
f(\tau \kappa+(1-\tau) \gamma, s u+(1-s) v) \leq \tau s f(\kappa, u)+\tau(1-s) f(\kappa, v)+s(1-\tau) f(\gamma, u)+(1-\tau)(1-s) f(\gamma, v) .
$$


The mapping $f$ is a co-ordinated concave on $\Delta$ if the inequality holds in reversed direction for all $\tau, s \in[0,1]$ and $(\kappa, u),(\gamma, v) \in \Delta$.

Definition 2. A function $f: \Delta=:[a, b] \times[c, d] \subset[0, \infty)^{2} \rightarrow \mathbb{R}$ is called $s$-Godunova -Levin in the second sense on the co-ordinates on $\Delta$ if

$$
f(\tau \kappa+(1-\tau) \gamma, \varsigma u+(1-\varsigma) v) \leq \frac{1}{\tau^{s} \varsigma^{s}} f(\kappa, u)+\frac{1}{\tau^{s}(1-\varsigma)^{s}} f(\kappa, v)+\frac{1}{\varsigma^{s}(1-\tau)^{s}} f(\gamma, u)+\frac{1}{(1-\tau)^{s}(1-\varsigma)^{s}} f(\gamma, v)
$$

holds for $\tau, \varsigma \in[0,1]$ and $(\kappa, u),(\gamma, v) \in \Delta$ with some $s \in(0,1]$.

Lemma 1. [37] Let $f: \Delta \rightarrow \mathbb{R}$ be a twice partially differentiable mapping on $\Delta^{\circ}$. If $\frac{\partial^{2} f}{\partial \tau \partial s} \in L(\Delta)$, then we have the following equality holds;

$$
\begin{aligned}
f(\kappa, \gamma) & +\frac{1}{(b-a)(d-c)} \int_{a}^{b} f(u, v) d v d u-\Phi=\frac{(\kappa-a)^{2}(\gamma-c)^{2}}{(b-a)(d-c)} \\
& \times \int_{0}^{1} \int_{0}^{1} \varsigma \tau \frac{\partial^{2} f}{\partial \varsigma \partial \tau}(\tau \kappa+(1-\tau) a, \varsigma \gamma+(1-\varsigma) c) d \varsigma d \tau-\frac{(\kappa-a)^{2}(d-\gamma)^{2}}{(b-a)(d-c)} \\
& \times \int_{0}^{1} \int_{0}^{1} \varsigma \tau \frac{\partial^{2} f}{\partial \varsigma \partial \tau}(\tau \kappa+(1-\tau) a, \varsigma \gamma+(1-\varsigma) d) d \varsigma d \tau-\frac{(b-\kappa)^{2}(\gamma-c)^{2}}{(b-a)(d-c)} \\
& \times \int_{0}^{1} \int_{0}^{1} \varsigma \tau \frac{\partial^{2} f}{\partial \varsigma \partial \tau}(\tau \kappa+(1-\tau) b, \varsigma \gamma+(1-\varsigma) c) d \varsigma d \tau+\frac{(b-\kappa)^{2}(d-\gamma)^{2}}{(b-a)(d-c)} \\
& \times \int_{0}^{1} \int_{0}^{1} \varsigma \tau \frac{\partial^{2} f}{\partial \varsigma \partial \tau}(\tau \kappa+(1-\tau) b, \varsigma \gamma+(1-\varsigma) d) d \varsigma d \tau
\end{aligned}
$$

for all $(\kappa, \gamma) \in \Delta$, where

$$
\Phi=\frac{1}{d-c} \int_{c}^{d} f(\kappa, v) d v+\frac{1}{b-a} \int_{a}^{b} f(u, \gamma) d u
$$

\section{Main results}

In this section, we present ostrowski type inequalities for co-ordinated $s$-Godunova-Levin convex functions.

Theorem 1. Let $\Delta=:[a, b] \times[c, d] \subset[0, \infty)^{2} \rightarrow \mathbb{R}$ be a twice partial differentiable mapping on $\Delta^{\circ}$ such that $\left|\frac{\partial^{2} f}{\partial s \partial \tau}\right| \in$ $L(\Delta)$. If $\left|\frac{\partial^{2} f}{\partial s \partial \tau}\right|$ is a co-ordinated s-Godunova-Levin convex on $\Delta$ with $s \in(0,1]$ and $(\kappa, \gamma) \in \Delta$, then we have the following inequality

$$
\begin{aligned}
& \left|f(\kappa, \gamma)+\frac{1}{(b-a)(d-c)} \int_{a}^{b} f(u, v) d v d u-\Phi\right| \leq \frac{(\kappa-a)^{2}(\gamma-c)^{2}}{(b-a)(d-c)}\left\{\left|\frac{\partial^{2} f}{\partial \varsigma \partial \tau} f(\kappa, \gamma)\right| \frac{1}{(2-s)^{2}}\right. \\
& \left.+\left|\frac{\partial^{2} f}{\partial \varsigma \partial \tau} f(\kappa, c)\right| \frac{1}{(1-s)(2-s)^{2}}+\left|\frac{\partial^{2} f}{\partial \varsigma \partial \tau} f(a, \gamma)\right| \frac{1}{(1-s)(2-s)^{2}}+\left|\frac{\partial^{2} f}{\partial \varsigma \partial \tau} f(a, c)\right| \frac{1}{(1-s)^{2}(2-s)^{2}}\right\} \\
& +\frac{(\kappa-a)^{2}(d-\gamma)^{2}}{(b-a)(d-c)}\left\{\left|\frac{\partial^{2} f}{\partial \varsigma \partial \tau} f(\kappa, \gamma)\right| \frac{1}{(2-s)^{2}}+\left|\frac{\partial^{2} f}{\partial \varsigma \partial \tau} f(\kappa, d)\right| \frac{1}{(1-s)(2-s)^{2}}\right.
\end{aligned}
$$




$$
\begin{aligned}
& \left.+\left|\frac{\partial^{2} f}{\partial \varsigma \partial \tau} f(a, \gamma)\right| \frac{1}{(1-s)(2-s)^{2}}+\left|\frac{\partial^{2} f}{\partial \varsigma \partial \tau} f(a, d)\right| \frac{1}{(1-s)^{2}(2-s)^{2}}\right\} \\
& +\frac{(b-\kappa)^{2}(\gamma-c)^{2}}{(b-a)(d-c)}\left\{\left|\frac{\partial^{2} f}{\partial \varsigma \partial \tau} f(\kappa, \gamma)\right| \frac{1}{(2-s)^{2}}+\left|\frac{\partial^{2} f}{\partial \varsigma \partial \tau} f(\kappa, c)\right| \frac{1}{(1-s)(2-s)^{2}}\right. \\
& \left.+\left|\frac{\partial^{2} f}{\partial \varsigma \partial \tau} f(b, \gamma)\right| \frac{1}{(1-s)(2-s)^{2}}+\left|\frac{\partial^{2} f}{\partial \varsigma \partial \tau} f(b, c)\right| \frac{1}{(1-s)^{2}(2-s)^{2}}\right\} \\
& +\frac{(b-\kappa)^{2}(d-\gamma)^{2}}{(b-a)(d-c)}\left\{\left|\frac{\partial^{2} f}{\partial \varsigma \partial \tau} f(\kappa, \gamma)\right| \frac{1}{(2-s)^{2}}+\left|\frac{\partial^{2} f}{\partial \varsigma \partial \tau} f(\kappa, d)\right| \frac{1}{(1-s)(2-s)^{2}}\right. \\
& \left.+\left|\frac{\partial^{2} f}{\partial \varsigma \partial \tau} f(b, \gamma)\right| \frac{1}{(1-s)(2-s)^{2}}+\left|\frac{\partial^{2} f}{\partial \varsigma \partial \tau} f(b, d)\right| \frac{1}{(1-s)^{2}(2-s)^{2}}\right\} .
\end{aligned}
$$

for all $(\kappa, \gamma) \in \Delta$, where $\Phi$ is defined in Lemma 1 .

Proof. From Lemma 1, we have

$$
\begin{aligned}
& \left|f(\kappa, \gamma)+\frac{1}{(b-a)(d-c)} \int_{a}^{b} f(u, v) d v d u-\Phi\right| \leq \frac{(\kappa-a)^{2}(\gamma-c)^{2}}{(b-a)(d-c)} \\
& \times \int_{0}^{1} \int_{0}^{1} \varsigma \tau\left|\frac{\partial^{2} f}{\partial \varsigma \partial \tau}(\tau \kappa+(1-\tau) a, \varsigma \gamma+(1-\varsigma) c)\right| d \varsigma d \tau+\frac{(\kappa-a)^{2}(d-\gamma)^{2}}{(b-a)(d-c)} \\
& \times \int_{0}^{1} \int_{0}^{1} \varsigma \tau\left|\frac{\partial^{2} f}{\partial \varsigma \partial \tau}(\tau \kappa+(1-\tau) a, \varsigma \gamma+(1-\varsigma) d)\right| d \varsigma d \tau+\frac{(b-\kappa)^{2}(\gamma-c)^{2}}{(b-a)(d-c)} \\
& \times \int_{0}^{1} \int_{0}^{1} \varsigma \tau\left|\frac{\partial^{2} f}{\partial \varsigma \partial \tau}(\tau \kappa+(1-\tau) b, \varsigma \gamma+(1-\varsigma) c)\right| d \varsigma d \tau+\frac{(b-\kappa)^{2}(d-\gamma)^{2}}{(b-a)(d-c)} \\
& \times \int_{0}^{1} \int_{0}^{1} \varsigma \tau\left|\frac{\partial^{2} f}{\partial \varsigma \partial \tau}(\tau \kappa+(1-\tau) b, \varsigma \gamma+(1-\varsigma) d)\right| d \varsigma d \tau
\end{aligned}
$$

Using the co-ordinated $s$-Godunova-Levin convexity of $\left|\frac{\partial^{2} f}{\partial \zeta \partial \tau}\right|$, we obtain that the following inequality holds

$$
\begin{aligned}
& \int_{0}^{1} \int_{0}^{1} \varsigma \tau\left|\frac{\partial^{2} f}{\partial \varsigma \partial \tau}(\tau \kappa+(1-\tau) a, \varsigma \gamma+(1-\varsigma) c)\right| d \varsigma d \tau \\
& \leq\left|\frac{\partial^{2} f}{\partial \varsigma \partial \tau} f(\kappa, \gamma)\right| \int_{0}^{1} \int_{0}^{1} \varsigma \tau \frac{1}{\tau^{s} \varsigma^{s}} d \varsigma d \tau+\left|\frac{\partial^{2} f}{\partial \varsigma \partial \tau} f(\kappa, c)\right| \int_{0}^{1} \int_{0}^{1} \varsigma \tau \frac{1}{\tau^{s}(1-\varsigma)^{s}} d \varsigma d \tau \\
& +\left|\frac{\partial^{2} f}{\partial \varsigma \partial \tau} f(a, \gamma)\right| \int_{0}^{1} \int_{0}^{1} \varsigma \tau \frac{1}{\varsigma^{s}(1-\tau)^{s}} d \varsigma d \tau+\left|\frac{\partial^{2} f}{\partial \varsigma \partial \tau} f(a, c)\right| \int_{0}^{1} \int_{0}^{1} \varsigma \tau \frac{1}{(1-\tau)^{s}(1-\varsigma)^{s}} d \varsigma d \tau .
\end{aligned}
$$

Since

$$
\begin{gathered}
\int_{0}^{1} \int_{0}^{1} \varsigma^{1-s} \tau^{1-s} d \varsigma d \tau=\frac{1}{(2-s)^{2}} \\
\int_{0}^{1} \int_{0}^{1} \varsigma(1-\varsigma)^{-s} \tau^{1-s} d \varsigma d \tau=\int_{0}^{1} \int_{0}^{1} \varsigma^{1-s} \tau(1-\tau)^{-s} d \varsigma d \tau=\frac{1}{(1-s)(2-s)^{2}}
\end{gathered}
$$

and

$$
\int_{0}^{1} \int_{0}^{1} \varsigma(1-\varsigma)^{-s} \tau(1-\tau)^{-s} d \varsigma d \tau=\frac{1}{(1-s)^{2}(2-s)^{2}}
$$


we have

$$
\begin{aligned}
H_{1} & =\int_{0}^{1} \int_{0}^{1} \varsigma \tau\left|\frac{\partial^{2} f}{\partial \varsigma \partial \tau}(\tau \kappa+(1-\tau) a, \varsigma \gamma+(1-\varsigma) c)\right| d \varsigma d \tau \\
& \leq\left|\frac{\partial^{2} f}{\partial \varsigma \partial \tau} f(\kappa, \gamma)\right| \frac{1}{(2-s)^{2}}+\left|\frac{\partial^{2} f}{\partial \varsigma \partial \tau} f(\kappa, c)\right| \frac{1}{(1-s)(2-s)^{2}} \\
& +\left|\frac{\partial^{2} f}{\partial \varsigma \partial \tau} f(a, \gamma)\right| \frac{1}{(1-s)(2-s)^{2}}+\left|\frac{\partial^{2} f}{\partial \varsigma \partial \tau} f(a, c)\right| \frac{1}{(1-s)^{2}(2-s)^{2}}
\end{aligned}
$$

Similarly, we get

$$
\begin{aligned}
H_{2} & =\int_{0}^{1} \int_{0}^{1} \varsigma \tau\left|\frac{\partial^{2} f}{\partial \varsigma \partial \tau}(\tau \kappa+(1-\tau) a, \varsigma \gamma+(1-\varsigma) d)\right| d \varsigma d \tau \\
& \leq\left|\frac{\partial^{2} f}{\partial \varsigma \partial \tau} f(\kappa, \gamma)\right| \frac{1}{(2-s)^{2}}+\left|\frac{\partial^{2} f}{\partial \varsigma \partial \tau} f(\kappa, d)\right| \frac{1}{(1-s)(2-s)^{2}} \\
& +\left|\frac{\partial^{2} f}{\partial \varsigma \partial \tau} f(a, \gamma)\right| \frac{1}{(1-s)(2-s)^{2}}+\left|\frac{\partial^{2} f}{\partial \varsigma \partial \tau} f(a, d)\right| \frac{1}{(1-s)^{2}(2-s)^{2}} \\
H_{3} & =\int_{0}^{1} \int_{0}^{1} \varsigma \tau\left|\frac{\partial^{2} f}{\partial \varsigma \partial \tau}(\tau \kappa+(1-\tau) b, \varsigma \gamma+(1-\varsigma) c)\right| d \varsigma d \tau \\
& \leq\left|\frac{\partial^{2} f}{\partial \varsigma \partial \tau} f(\kappa, \gamma)\right| \frac{1}{(2-s)^{2}}+\left|\frac{\partial^{2} f}{\partial \varsigma \partial \tau} f(\kappa, c)\right| \frac{1}{(1-s)(2-s)^{2}} \\
& +\left|\frac{\partial^{2} f}{\partial \varsigma \partial \tau} f(b, \gamma)\right| \frac{1}{(1-s)(2-s)^{2}}+\left|\frac{\partial^{2} f}{\partial \varsigma \partial \tau} f(b, c)\right| \frac{1}{(1-s)^{2}(2-s)^{2}}
\end{aligned}
$$

and

$$
\begin{aligned}
H_{4} & =\int_{0}^{1} \int_{0}^{1} \varsigma \tau\left|\frac{\partial^{2} f}{\partial \varsigma \partial \tau}(\tau \kappa+(1-\tau) b, \varsigma \gamma+(1-\varsigma) d)\right| d \varsigma d \tau \\
& \leq\left|\frac{\partial^{2} f}{\partial \varsigma \partial \tau} f(\kappa, \gamma)\right| \frac{1}{(2-s)^{2}}+\left|\frac{\partial^{2} f}{\partial \varsigma \partial \tau} f(\kappa, d)\right| \frac{1}{(1-s)(2-s)^{2}} \\
& +\left|\frac{\partial^{2} f}{\partial \varsigma \partial \tau} f(b, \gamma)\right| \frac{1}{(1-s)(2-s)^{2}}+\left|\frac{\partial^{2} f}{\partial \varsigma \partial \tau} f(b, d)\right| \frac{1}{(1-s)^{2}(2-s)^{2}}
\end{aligned}
$$

The proof is completed.

Corollary 1. Under assumptions of Theorem 1 with $\kappa=\frac{a+b}{2}$ and $\gamma=\frac{c+d}{2}$, we have the following inequality holds:

$$
\begin{aligned}
& \left|f(\kappa, \gamma)+\frac{1}{(b-a)(d-c)} \int_{a}^{b} f(u, v) d v d u-\Phi\right| \leq \frac{(b-a)(d-c)}{(2-s)^{2}}\left|\frac{\partial^{2} f}{\partial \varsigma \partial \tau} f\left(\frac{a+b}{2}, \frac{c+d}{2}\right)\right| \\
& +\frac{(b-a)(d-c)}{2(1-s)(2-s)^{2}}\left\{\left|\frac{\partial^{2} f}{\partial \varsigma \partial \tau} f\left(\frac{a+b}{2}, c\right)\right|+\left|\frac{\partial^{2} f}{\partial \varsigma \partial \tau} f\left(\frac{a+b}{2}, d\right)\right|+\left|\frac{\partial^{2} f}{\partial \varsigma \partial \tau} f\left(a, \frac{c+d}{2}\right)\right|+\left|\frac{\partial^{2} f}{\partial \varsigma \partial \tau} f\left(b, \frac{c+d}{2}\right)\right|\right\} \\
& +\frac{(b-a)(d-c)}{4(1-s)^{2}(2-s)^{2}}\left\{\left|\frac{\partial^{2} f}{\partial \varsigma \partial \tau} f(a, c)\right|+\left|\frac{\partial^{2} f}{\partial \varsigma \partial \tau} f(a, d)\right|+\left|\frac{\partial^{2} f}{\partial \varsigma \partial \tau} f(b, d)\right|+\left|\frac{\partial^{2} f}{\partial \varsigma \partial \tau} f(b, c)\right|\right\} .
\end{aligned}
$$


Theorem 2. Let $\Delta=:[a, b] \times[c, d] \subset[0, \infty)^{2} \rightarrow \mathbb{R}$ be a twice partial differentiable mapping on $\Delta^{\circ}$ such that $\left|\frac{\partial^{2} f}{\partial s \partial \tau}\right| \in$ $L(\Delta)$. If $\left|\frac{\partial^{2} f}{\partial s \partial \tau}\right|^{q}, q>1$, is a co-ordinated s-Godunova-Levin convex on $\Delta$ with $s \in(0,1]$ and $(\kappa, \gamma) \in \Delta$, then we have the following inequality holds

$$
\begin{aligned}
& \left|f(\kappa, \gamma)+\frac{1}{(b-a)(d-c)} \int_{a}^{b} f(u, v) d v d u-\Phi\right| \\
& \leq \frac{1}{(1+p)^{\frac{2}{p}}} \times\left\{\frac{(\kappa-a)^{2}(\gamma-c)^{2}}{(b-a)(d-c)} \frac{1}{(1-s)^{2}}\left(\left|\frac{\partial^{2} f}{\partial \varsigma \partial \tau} f(\kappa, \gamma)\right|^{q}+\left|\frac{\partial^{2} f}{\partial \varsigma \partial \tau} f(\kappa, c)\right|^{q}+\left|\frac{\partial^{2} f}{\partial \varsigma \partial \tau} f(a, \gamma)\right|^{q}+\left|\frac{\partial^{2} f}{\partial \varsigma \partial \tau} f(a, c)\right|^{q}\right)^{\frac{1}{q}}\right. \\
& +\frac{(\kappa-a)^{2}(d-\gamma)^{2}}{(b-a)(d-c)} \frac{1}{(1-s)^{2}}\left(\left|\frac{\partial^{2} f}{\partial \varsigma \partial \tau} f(\kappa, \gamma)\right|^{q}+\left|\frac{\partial^{2} f}{\partial \varsigma \partial \tau} f(\kappa, d)\right|^{q}+\left|\frac{\partial^{2} f}{\partial \varsigma \partial \tau} f(a, \gamma)\right|^{q}+\left|\frac{\partial^{2} f}{\partial \varsigma \partial \tau} f(a, d)\right|^{q}\right)^{\frac{1}{q}} \\
& +\frac{(b-\kappa)^{2}(\gamma-c)^{2}}{(b-a)(d-c)} \frac{1}{(1-s)^{2}}\left(\left|\frac{\partial^{2} f}{\partial \varsigma \partial \tau} f(\kappa, \gamma)\right|^{q}+\left|\frac{\partial^{2} f}{\partial \varsigma \partial \tau} f(\kappa, c)\right|^{q}+\left|\frac{\partial^{2} f}{\partial \varsigma \partial \tau} f(b, \gamma)\right|^{q}+\left|\frac{\partial^{2} f}{\partial \varsigma \partial \tau} f(b, c)\right|^{q}\right)^{\frac{1}{q}} \\
& \left.+\frac{(b-\kappa)^{2}(d-\gamma)^{2}}{(b-a)(d-c)} \frac{1}{(1-s)^{2}}\left(\left|\frac{\partial^{2} f}{\partial \varsigma \partial \tau} f(\kappa, \gamma)\right|^{q}+\left|\frac{\partial^{2} f}{\partial \varsigma \partial \tau} f(\kappa, d)\right|^{q}+\left|\frac{\partial^{2} f}{\partial \varsigma \partial \tau} f(b, \gamma)\right|^{q}+\left|\frac{\partial^{2} f}{\partial \varsigma \partial \tau} f(b, d)\right|^{q}\right)^{\frac{1}{q}}\right\}
\end{aligned}
$$

$\frac{1}{p}+\frac{1}{q}=1$. For all $(\kappa, \gamma) \in \Delta$, where $\Phi$ is defined in Lemma 1.

Proof. From Lemma 1 and using the Hölder inequality for double integrals, we have that

$$
\begin{aligned}
& \left|f(\kappa, \gamma)+\frac{1}{(b-a)(d-c)} \int_{a}^{b} f(u, v) d v d u-\Phi\right| \\
& \leq\left(\int_{0}^{1} \int_{0}^{1} \varsigma^{p} \tau^{p}\right)^{\frac{1}{p}} \times\left\{\frac{(\kappa-a)^{2}(\gamma-c)^{2}}{(b-a)(d-c)}\left(\int_{0}^{1} \int_{0}^{1}\left|\frac{\partial^{2} f}{\partial \varsigma \partial \tau}(\tau \kappa+(1-\tau) a, \varsigma \gamma+(1-\varsigma) c)\right|^{q} d \varsigma d \tau\right)^{\frac{1}{q}}\right. \\
& +\frac{(\kappa-a)^{2}(d-\gamma)^{2}}{(b-a)(d-c)}\left(\int_{0}^{1} \int_{0}^{1}\left|\frac{\partial^{2} f}{\partial \varsigma \partial \tau}(\tau \kappa+(1-\tau) a, \varsigma \gamma+(1-\varsigma) d)\right|^{q} d \varsigma d \tau\right)^{\frac{1}{q}} \\
& +\frac{(b-\kappa)^{2}(\gamma-c)^{2}}{(b-a)(d-c)}\left(\int_{0}^{1} \int_{0}^{1}\left|\frac{\partial^{2} f}{\partial \varsigma \partial \tau}(\tau \kappa+(1-\tau) b, \varsigma \gamma+(1-\varsigma) c)\right|^{q} d \varsigma d \tau\right)^{\frac{1}{q}} \\
& \left.+\frac{(b-\kappa)^{2}(d-\gamma)^{2}}{(b-a)(d-c)}\left(\int_{0}^{1} \int_{0}^{1}\left|\frac{\partial^{2} f}{\partial \varsigma \partial \tau}(\tau \kappa+(1-\tau) b, \varsigma \gamma+(1-\varsigma) d)\right|^{q} d \varsigma d \tau\right)^{\frac{1}{q}}\right\}
\end{aligned}
$$

for all $(\kappa, \gamma) \in \Delta$

Using the co-ordinated s-Godunova-Levin convexity of $\left|\frac{\partial^{2} f}{\partial \varsigma \partial \tau}\right|^{q}$, we obtain that the following inequality holds

$$
\begin{aligned}
& \int_{0}^{1} \int_{0}^{1}\left|\frac{\partial^{2} f}{\partial \varsigma \partial \tau}(\tau \kappa+(1-\tau) a, \varsigma \gamma+(1-\varsigma) c)\right|^{q} d \varsigma d \tau \\
& \leq\left|\frac{\partial^{2} f}{\partial \varsigma \partial \tau} f(\kappa, \gamma)\right|^{q} \int_{0}^{1} \int_{0}^{1} \varsigma^{-s} \tau^{-s} d \varsigma d \tau+\left|\frac{\partial^{2} f}{\partial \varsigma \partial \tau} f(\kappa, c)\right|^{q} \int_{0}^{1} \int_{0}^{1} \tau^{-s}(1-\varsigma)^{-s} d \varsigma d \tau \\
& +\left|\frac{\partial^{2} f}{\partial \varsigma \partial \tau} f(a, \gamma)\right|^{q} \int_{0}^{1} \int_{0}^{1} \varsigma^{-s}(1-\tau)^{-s} d \varsigma d \tau+\left|\frac{\partial^{2} f}{\partial \varsigma \partial \tau} f(a, c)\right|^{q} \int_{0}^{1} \int_{0}^{1}(1-\varsigma)^{-s}(1-\tau)^{-s} d \varsigma d \tau
\end{aligned}
$$




$$
=\frac{1}{(1-s)^{2}}\left\{\left|\frac{\partial^{2} f}{\partial \varsigma \partial \tau} f(\kappa, \gamma)\right|^{q}+\left|\frac{\partial^{2} f}{\partial \varsigma \partial \tau} f(\kappa, c)\right|^{q}+\left|\frac{\partial^{2} f}{\partial \varsigma \partial \tau} f(a, \gamma)\right|^{q}+\left|\frac{\partial^{2} f}{\partial \varsigma \partial \tau} f(a, c)\right|^{q}\right\}
$$

Similarly, we also have the following inequality

$$
\begin{aligned}
& \int_{0}^{1} \int_{0}^{1}\left|\frac{\partial^{2} f}{\partial \varsigma \partial \tau}(\tau \kappa+(1-\tau) a, \varsigma \gamma+(1-\varsigma) d)\right|^{q} d \varsigma d \tau \\
& \leq \frac{1}{(1-s)^{2}}\left\{\left|\frac{\partial^{2} f}{\partial \varsigma \partial \tau} f(\kappa, \gamma)\right|^{q}+\left|\frac{\partial^{2} f}{\partial \varsigma \partial \tau} f(\kappa, d)\right|^{q}+\left|\frac{\partial^{2} f}{\partial \varsigma \partial \tau} f(a, \gamma)\right|^{q}+\left|\frac{\partial^{2} f}{\partial \varsigma \partial \tau} f(a, d)\right|^{q}\right\} \\
& \int_{0}^{1} \int_{0}^{1}\left|\frac{\partial^{2} f}{\partial \varsigma \partial \tau}(\tau \kappa+(1-\tau) b, \varsigma \gamma+(1-\varsigma) c)\right|^{q} d \varsigma d \tau \\
& \leq \frac{1}{(1-s)^{2}}\left\{\left|\frac{\partial^{2} f}{\partial \varsigma \partial \tau} f(\kappa, \gamma)\right|^{q}+\left|\frac{\partial^{2} f}{\partial \varsigma \partial \tau} f(\kappa, c)\right|^{q}+\left|\frac{\partial^{2} f}{\partial \varsigma \partial \tau} f(b, \gamma)\right|^{q}+\left|\frac{\partial^{2} f}{\partial \varsigma \partial \tau} f(b, c)\right|^{q}\right\}
\end{aligned}
$$

and

$$
\begin{aligned}
& \int_{0}^{1} \int_{0}^{1}\left|\frac{\partial^{2} f}{\partial \varsigma \partial \tau}(\tau \kappa+(1-\tau) b, \varsigma \gamma+(1-\varsigma) d)\right|^{q} d \varsigma d \tau \\
& \leq \frac{1}{(1-s)^{2}}\left\{\left|\frac{\partial^{2} f}{\partial \varsigma \partial \tau} f(\kappa, \gamma)\right|^{q}+\left|\frac{\partial^{2} f}{\partial \varsigma \partial \tau} f(\kappa, d)\right|^{q}+\left|\frac{\partial^{2} f}{\partial \varsigma \partial \tau} f(b, \gamma)\right|^{q}+\left|\frac{\partial^{2} f}{\partial \varsigma \partial \tau} f(b, d)\right|^{q}\right\} .
\end{aligned}
$$

Using the fact that

$$
\left(\int_{0}^{1} \int_{0}^{1} \varsigma^{p} \tau^{p} d \varsigma d \tau\right)^{\frac{1}{p}}=\frac{1}{(1+p)^{\frac{2}{p}}}
$$

and the above inequalities in (4), we get (3). This completes the proof of the theorem.

Corollary 2. Under assumptions of Theorem 2 with $\kappa=\frac{a+b}{2}$ and $\gamma=\frac{c+d}{2}$, we have the following inequality holds:

$$
\begin{aligned}
& \left|f(\kappa, \gamma)+\frac{1}{(b-a)(d-c)} \int_{a}^{b} f(u, v) d v d u-\Phi\right| \leq \frac{1}{(1+p)^{\frac{2}{p}}} \mid \begin{array}{l}
(b-a)(d-c) \\
4(1-s)^{2}
\end{array}\left(\left|\frac{\partial^{2} f}{\partial \varsigma \partial \tau} f\left(\frac{a+b}{2}, \frac{c+d}{2}\right)\right|^{q}+\left|\frac{\partial^{2} f}{\partial \varsigma \partial \tau} f\left(\frac{a+b}{2}, c\right)\right|^{q}+\left|\frac{\partial^{2} f}{\partial \varsigma \partial \tau} f\left(a, \frac{c+d}{2}\right)\right|^{q}+\left|\frac{\partial^{2} f}{\partial \varsigma \partial \tau} f(a, c)\right|^{q}\right)^{\frac{1}{q}} \\
& +\frac{(b-a)(d-c)}{4(1-s)^{2}}\left(\left|\frac{\partial^{2} f}{\partial \varsigma \partial \tau} f\left(\frac{a+b}{2}, \frac{c+d}{2}\right)\right|^{q}+\left|\frac{\partial^{2} f}{\partial \varsigma \partial \tau} f\left(\frac{a+b}{2}, d\right)\right|^{q}+\left|\frac{\partial^{2} f}{\partial \varsigma \partial \tau} f\left(a, \frac{c+d}{2}\right)\right|^{q}+\left|\frac{\partial^{2} f}{\partial \varsigma \partial \tau} f(a, d)\right|^{q}\right)^{\frac{1}{q}} \\
& +\frac{(b-a)(d-c)}{4(1-s)^{2}}\left(\left|\frac{\partial^{2} f}{\partial \varsigma \partial \tau} f\left(\frac{a+b}{2}, \frac{c+d}{2}\right)\right|^{q}+\left|\frac{\partial^{2} f}{\partial \varsigma \partial \tau} f\left(\frac{a+b}{2}, c\right)\right|^{q}+\left|\frac{\partial^{2} f}{\partial \varsigma \partial \tau} f\left(b, \frac{c+d}{2}\right)\right|^{q}+\left|\frac{\partial^{2} f}{\partial \varsigma \partial \tau} f(b, c)\right|^{q}\right)^{\frac{1}{q}} \\
& \left.+\frac{(b-a)(d-c)}{4(1-s)^{2}}\left(\left|\frac{\partial^{2} f}{\partial \varsigma \partial \tau} f\left(\frac{a+b}{2}, \frac{c+d}{2}\right)\right|^{q}+\left|\frac{\partial^{2} f}{\partial \varsigma \partial \tau} f\left(\frac{a+b}{2}, d\right)\right|^{q}+\left|\frac{\partial^{2} f}{\partial \varsigma \partial \tau} f\left(b, \frac{c+d}{2}\right)\right|^{q}+\left|\frac{\partial^{2} f}{\partial \varsigma \partial \tau} f(b, d)\right|^{q}\right)^{\frac{1}{q}}\right\} .
\end{aligned}
$$

Theorem 3. Let $\Delta=:[a, b] \times[c, d] \subset[0, \infty)^{2} \rightarrow \mathbb{R}$ be a twice partial differentiable mapping on $\Delta^{\circ}$ such that $\left|\frac{\partial^{2} f}{\partial s \partial \tau}\right| \in$ $L(\Delta)$. If $\left|\frac{\partial^{2} f}{\partial s \partial \tau}\right|^{q}, q>1$, is a co-ordinated s-Godunova-Levin convex on $\Delta$ with $s \in(0,1]$ and $(\kappa, \gamma) \in \Delta$, then we have 
the following inequality

$$
\begin{aligned}
& \left|f(\kappa, \gamma)+\frac{1}{(b-a)(d-c)} \int_{a}^{b} f(u, v) d v d u-\Phi\right| \leq\left(\frac{1}{4}\right)^{1-\frac{1}{q}} \\
& \times\left\{\frac { ( \kappa - a ) ^ { 2 } ( \gamma - c ) ^ { 2 } } { ( b - a ) ( d - c ) } \left(\left|\frac{\partial^{2} f}{\partial \varsigma \partial \tau} f(\kappa, \gamma)\right|^{q} \frac{1}{(2-s)^{2}}+\left|\frac{\partial^{2} f}{\partial \varsigma \partial \tau} f(\kappa, c)\right|^{q} \frac{1}{(1-s)(2-s)^{2}}\right.\right. \\
& \left.+\left|\frac{\partial^{2} f}{\partial \varsigma \partial \tau} f(a, \gamma)\right|^{q} \frac{1}{(1-s)(2-s)^{2}}+\left|\frac{\partial^{2} f}{\partial \varsigma \partial \tau} f(a, c)\right|^{q} \frac{1}{(1-s)^{2}(2-s)^{2}}\right)^{\frac{1}{q}} \\
& +\frac{(\kappa-a)^{2}(d-\gamma)^{2}}{(b-a)(d-c)}\left(\left|\frac{\partial^{2} f}{\partial \varsigma \partial \tau} f(\kappa, \gamma)\right|^{q} \frac{1}{(2-s)^{2}}+\left|\frac{\partial^{2} f}{\partial \varsigma \partial \tau} f(\kappa, d)\right|^{q} \frac{1}{(1-s)(2-s)^{2}}\right. \\
& \left.+\left|\frac{\partial^{2} f}{\partial \varsigma \partial \tau} f(a, \gamma)\right|^{q} \frac{1}{(1-s)(2-s)^{2}}+\left|\frac{\partial^{2} f}{\partial \varsigma \partial \tau} f(a, d)\right|^{q} \frac{1}{(1-s)^{2}(2-s)^{2}}\right)^{\frac{1}{q}} \\
& +\frac{(b-\kappa)^{2}(\gamma-c)^{2}}{(b-a)(d-c)}\left(\left|\frac{\partial^{2} f}{\partial \varsigma \partial \tau} f(\kappa, \gamma)\right|^{q} \frac{1}{(2-s)^{2}}+\left|\frac{\partial^{2} f}{\partial \varsigma \partial \tau} f(\kappa, c)\right|^{q} \frac{1}{(1-s)(2-s)^{2}}\right. \\
& \left.+\left|\frac{\partial^{2} f}{\partial \varsigma \partial \tau} f(b, \gamma)\right|^{q} \frac{1}{(1-s)(2-s)^{2}}+\left|\frac{\partial^{2} f}{\partial \varsigma \partial \tau} f(b, c)\right|^{q} \frac{1}{(1-s)^{2}(2-s)^{2}}\right)^{\frac{1}{q}} \\
& +\frac{(b-\kappa)^{2}(d-\gamma)^{2}}{(b-a)(d-c)}\left(\left|\frac{\partial^{2} f}{\partial \varsigma \partial \tau} f(\kappa, \gamma)\right|^{q} \frac{1}{(2-s)^{2}}+\left|\frac{\partial^{2} f}{\partial \varsigma \partial \tau} f(\kappa, d)\right|^{q} \frac{1}{(1-s)(2-s)^{2}}\right. \\
& +\left|\frac{\partial^{2} f}{\partial \varsigma \partial} f(b, \gamma)\right|^{\frac{1}{q}} \\
& \left.+\frac{1}{(1-s)(2-s)^{2}}+\mid \frac{1}{(1-s)^{2}(2-s)^{2}}\right)^{q} f(b, d)
\end{aligned}
$$

for all $(\kappa, \gamma) \in \Delta$, where $\Phi$ is defined in Lemma 1 .

Proof. From Lemma 1 and using the power mean inequality for double integrals, we have

$$
\begin{aligned}
& \left|f(\kappa, \gamma)+\frac{1}{(b-a)(d-c)} \int_{a}^{b} f(u, v) d v d u-\Phi\right| \leq\left(\int_{0}^{1} \int_{0}^{1} \varsigma \tau\right)^{1-\frac{1}{q}} \\
& \times\left\{\frac{(\kappa-a)^{2}(\gamma-c)^{2}}{(b-a)(d-c)}\left(\int_{0}^{1} \int_{0}^{1} \varsigma \tau\left|\frac{\partial^{2} f}{\partial \varsigma \partial \tau}(\tau \kappa+(1-\tau) a, \varsigma \gamma+(1-\varsigma) c)\right|^{q} d \varsigma d \tau\right)^{\frac{1}{q}}\right. \\
& +\frac{(\kappa-a)^{2}(d-\gamma)^{2}}{(b-a)(d-c)}\left(\int_{0}^{1} \int_{0}^{1} \varsigma \tau\left|\frac{\partial^{2} f}{\partial \varsigma \partial \tau}(\tau \kappa+(1-\tau) a, \varsigma \gamma+(1-\varsigma) d)\right|^{q} d \varsigma d \tau\right)^{\frac{1}{q}} \\
& +\frac{(b-\kappa)^{2}(\gamma-c)^{2}}{(b-a)(d-c)}\left(\int_{0}^{1} \int_{0}^{1} \varsigma \tau\left|\frac{\partial^{2} f}{\partial \varsigma \partial \tau}(\tau \kappa+(1-\tau) b, \varsigma \gamma+(1-\varsigma) c)\right|^{q} d \varsigma d \tau\right)^{\frac{1}{q}} \\
& \left.+\frac{(b-\kappa)^{2}(d-\gamma)^{2}}{(b-a)(d-c)}\left(\int_{0}^{1} \int_{0}^{1} \varsigma \tau\left|\frac{\partial^{2} f}{\partial \varsigma \partial \tau}(\tau \kappa+(1-\tau) b, \varsigma \gamma+(1-\varsigma) d)\right|^{q} d \varsigma d \tau\right)^{\frac{1}{q}}\right\}
\end{aligned}
$$

Using the co-ordinated $s$-Godunova-Levin convexity of $\left|\frac{\partial^{2} f}{\partial \varsigma \partial \tau}\right|^{q}$, we obtain that the following inequality holds 


$$
\begin{aligned}
& \int_{0}^{1} \int_{0}^{1} \varsigma \tau\left|\frac{\partial^{2} f}{\partial \varsigma \partial \tau}(\tau \kappa+(1-\tau) a, \varsigma \gamma+(1-\varsigma) c)\right|^{q} d \varsigma d \tau \\
& \leq\left|\frac{\partial^{2} f}{\partial \varsigma \partial \tau} f(\kappa, \gamma)\right|_{0}^{q} \int_{0}^{1} \int_{0}^{1} \varsigma^{1-s} \tau^{1-s} d \varsigma d \tau+\left|\frac{\partial^{2} f}{\partial \varsigma \partial \tau} f(\kappa, c)\right|^{q} \int_{0}^{1} \int_{0}^{1} \varsigma \tau^{1-s}(1-\varsigma)^{-s} d \varsigma d \tau \\
& +\left|\frac{\partial^{2} f}{\partial \varsigma \partial \tau} f(a, \gamma)\right|_{0}^{q} \int_{0}^{1} \int_{0}^{1} \tau \varsigma^{1-s}(1-\tau)^{-s} d \varsigma d \tau+\left|\frac{\partial^{2} f}{\partial \varsigma \partial \tau} f(a, c)\right|^{q} \int_{0}^{1} \int_{0}^{1} \varsigma \tau(1-\varsigma)^{-s}(1-\tau)^{-s} d \varsigma d \tau \\
& =\frac{1}{(2-s)^{2}}\left|\frac{\partial^{2} f}{\partial \varsigma \partial \tau} f(\kappa, \gamma)\right|^{q}+\frac{1}{(1-s)(2-s)^{2}}\left|\frac{\partial^{2} f}{\partial \varsigma \partial \tau} f(\kappa, c)\right|^{q} \\
& +\frac{1}{(1-s)(2-s)^{2}}\left|\frac{\partial^{2} f}{\partial \varsigma \partial \tau} f(a, \gamma)\right|^{q}+\frac{1}{(1-s)^{2}(2-s)^{2}}\left|\frac{\partial^{2} f}{\partial \varsigma \partial \tau} f(a, c)\right|^{q} . \\
& R_{1}=\int_{0}^{1} \int_{0}^{1} \varsigma \tau\left|\frac{\partial^{2} f}{\partial \varsigma \partial \tau}(\tau \kappa+(1-\tau) a, \varsigma \gamma+(1-\varsigma) c)\right| d \varsigma d \tau \\
& \leq\left|\frac{\partial^{2} f}{\partial \varsigma \partial \tau} f(\kappa, \gamma)\right|^{q} \frac{1}{(2-s)^{2}}+\left|\frac{\partial^{2} f}{\partial \varsigma \partial \tau} f(\kappa, c)\right|^{q} \frac{1}{(1-s)(2-s)^{2}} \\
& +\left|\frac{\partial^{2} f}{\partial \varsigma \partial \tau} f(a, \gamma)\right|^{q} \frac{1}{(1-s)(2-s)^{2}}+\left|\frac{\partial^{2} f}{\partial \varsigma \partial \tau} f(a, c)\right|^{q} \frac{1}{(1-s)^{2}(2-s)^{2}}
\end{aligned}
$$

Similarly, we get

$$
\begin{aligned}
R_{2} & =\int_{0}^{1} \int_{0}^{1} \varsigma \tau\left|\frac{\partial^{2} f}{\partial \varsigma \partial \tau}(\tau \kappa+(1-\tau) a, \varsigma \gamma+(1-\varsigma) d)\right| d \varsigma d \tau \\
& \leq\left|\frac{\partial^{2} f}{\partial \varsigma \partial \tau} f(\kappa, \gamma)\right|^{q} \frac{1}{(2-s)^{2}}+\left|\frac{\partial^{2} f}{\partial \varsigma \partial \tau} f(\kappa, d)\right|^{q} \frac{1}{(1-s)(2-s)^{2}} \\
& +\left|\frac{\partial^{2} f}{\partial \varsigma \partial \tau} f(a, \gamma)\right|^{q} \frac{1}{(1-s)(2-s)^{2}}+\left|\frac{\partial^{2} f}{\partial \varsigma \partial \tau} f(a, d)\right|^{q} \frac{1}{(1-s)^{2}(2-s)^{2}} \\
R_{3} & =\int_{0}^{1} \int_{0}^{1} \varsigma \tau\left|\frac{\partial^{2} f}{\partial \varsigma \partial \tau}(\tau \kappa+(1-\tau) b, \varsigma \gamma+(1-\varsigma) c)\right| d \varsigma d \tau \\
& \leq\left|\frac{\partial^{2} f}{\partial \varsigma \partial \tau} f(\kappa, \gamma)\right|^{q} \frac{1}{(2-s)^{2}}+\left|\frac{\partial^{2} f}{\partial \varsigma \partial \tau} f(\kappa, c)\right|^{q} \frac{1}{(1-s)(2-s)^{2}} \\
& +\left|\frac{\partial^{2} f}{\partial \varsigma \partial \tau} f(b, \gamma)\right|^{q} \frac{1}{(1-s)(2-s)^{2}}+\left|\frac{\partial^{2} f}{\partial \varsigma \partial \tau} f(b, c)\right|^{q} \frac{1}{(1-s)^{2}(2-s)^{2}}
\end{aligned}
$$

and

$$
\begin{aligned}
R_{4} & =\int_{0}^{1} \int_{0}^{1} \varsigma \tau\left|\frac{\partial^{2} f}{\partial \varsigma \partial \tau}(\tau \kappa+(1-\tau) b, \varsigma \gamma+(1-\varsigma) d)\right| d \varsigma d \tau \\
& \leq\left|\frac{\partial^{2} f}{\partial \varsigma \partial \tau} f(\kappa, \gamma)\right|^{q} \frac{1}{(2-s)^{2}}+\left|\frac{\partial^{2} f}{\partial \varsigma \partial \tau} f(\kappa, d)\right|^{q} \frac{1}{(1-s)(2-s)^{2}} \\
& +\left|\frac{\partial^{2} f}{\partial \varsigma \partial \tau} f(b, \gamma)\right|^{q} \frac{1}{(1-s)(2-s)^{2}}+\left|\frac{\partial^{2} f}{\partial \varsigma \partial \tau} f(b, d)\right|^{q} \frac{1}{(1-s)^{2}(2-s)^{2}}
\end{aligned}
$$


Using the fact that

$$
\left(\int_{0}^{1} \int_{0}^{1} \varsigma \tau d \varsigma d \tau\right)^{1-\frac{1}{q}}=\left(\frac{1}{4}\right)^{1-\frac{1}{q}}
$$

and the above inequalities in (6), we get (5). This completes the proof of the theorem.

Corollary 3. Under assumptions of Theorem 3 with $\kappa=\frac{a+b}{2}$ and $\gamma=\frac{c+d}{2}$, we have the following inequality

$$
\begin{aligned}
& \left|f(\kappa, \gamma)+\frac{1}{(b-a)(d-c)} \int_{a}^{b} f(u, v) d v d u-\Phi\right| \leq\left(\frac{1}{4}\right)^{1-\frac{1}{q}} \\
& \times\left\{\frac { ( b - a ) ( d - c ) } { 4 } \left(\left|\frac{\partial^{2} f}{\partial \varsigma \partial \tau} f\left(\frac{a+b}{2}, \frac{c+d}{2}\right)\right|^{q} \frac{1}{(2-s)^{2}}+\left|\frac{\partial^{2} f}{\partial \varsigma \partial \tau} f\left(\frac{a+b}{2}, c\right)\right|^{q} \frac{1}{(1-s)(2-s)^{2}}\right.\right. \\
& \left.+\left|\frac{\partial^{2} f}{\partial \varsigma \partial \tau} f\left(a, \frac{c+d}{2}\right)\right|^{q} \frac{1}{(1-s)(2-s)^{2}}+\left|\frac{\partial^{2} f}{\partial \varsigma \partial \tau} f(a, c)\right|^{q} \frac{1}{(1-s)^{2}(2-s)^{2}}\right)^{\frac{1}{q}} \\
& +\frac{(b-a)(d-c)}{4}\left(\left|\frac{\partial^{2} f}{\partial \varsigma \partial \tau} f\left(\frac{a+b}{2}, \frac{c+d}{2}\right)\right|^{q} \frac{1}{(2-s)^{2}}+\left|\frac{\partial^{2} f}{\partial \varsigma \partial \tau} f\left(\frac{a+b}{2}, d\right)\right|^{q} \frac{1}{(1-s)(2-s)^{2}}\right. \\
& \left.+\left|\frac{\partial^{2} f}{\partial \varsigma \partial \tau} f\left(a, \frac{c+d}{2}\right)\right|^{q} \frac{1}{(1-s)(2-s)^{2}}+\left|\frac{\partial^{2} f}{\partial \varsigma \partial \tau} f(a, d)\right|^{q} \frac{1}{(1-s)^{2}(2-s)^{2}}\right)^{\frac{1}{q}} \\
& +\frac{(b-a)(d-c)}{4}\left(\left|\frac{\partial^{2} f}{\partial \varsigma \partial \tau} f\left(\frac{a+b}{2}, \frac{c+d}{2}\right)\right|^{q} \frac{1}{(2-s)^{2}}+\left|\frac{\partial^{2} f}{\partial \varsigma \partial \tau} f\left(\frac{a+b}{2}, c\right)\right|^{q} \frac{1}{(1-s)(2-s)^{2}}\right. \\
& \left.+\left|\frac{\partial^{2} f}{\partial \varsigma \partial \tau} f\left(b, \frac{c+d}{2}\right)\right|^{q} \frac{1}{(1-s)(2-s)^{2}}+\left|\frac{\partial^{2} f}{\partial \varsigma \partial \tau} f(b, c)\right|^{q} \frac{1}{(1-s)^{2}(2-s)^{2}}\right)^{\frac{1}{q}} \\
& +\frac{(b-a)(d-c)}{4}\left(\left|\frac{\partial^{2} f}{\partial \varsigma \partial \tau} f\left(\frac{a+b}{2}, \frac{c+d}{2}\right)\right|^{q} \frac{1}{(2-s)^{2}}+\left|\frac{\partial^{2} f}{\partial \varsigma \partial \tau} f\left(\frac{a+b}{2}, d\right)\right|^{q} \frac{1}{(1-s)(2-s)^{2}}\right. \\
& \left.\left.+\left|\frac{\partial^{2} f}{\partial \varsigma \partial \tau} f\left(b, \frac{c+d}{2}\right)\right|^{q} \frac{1}{(1-s)(2-s)^{2}}+\left|\frac{\partial^{2} f}{\partial \varsigma \partial \tau} f(b, d)\right|^{q} \frac{1}{(1-s)^{2}(2-s)^{2}}\right)^{\frac{1}{q}}\right\} \text {. }
\end{aligned}
$$

\section{Competing interests}

The authors declare that they have no competing interests.

\section{Authors' contributions}

All authors have contributed to all parts of the article. All authors read and approved the final manuscript.

\section{References}

[1] Ostrowski, A.M., Über die Absolutabweichung einer differentiebaren Function von ihrem integral mittelwert Commentarii Mathematici Helvetici 10 (1938), 226-227. 
[2] Grüss, G., Uber das maximum des absoluten Betrages von

$$
\frac{1}{b-a} \int_{a}^{b} f(\kappa) g(\kappa) d \kappa-\frac{1}{(b-a)^{2}} \int_{a}^{b} f(\kappa) d \kappa \int_{a}^{b} g(\kappa) d \kappa
$$

Math. Z 39 (1935) 215-226.

[3] Korkine A.N., Sur une therome de M. Tchebychef, C.r. Acad. Sci. Paris 96 (1883), 316-327.

[4] Hu Yue, Ostrowski Inequality for Fractional Integrals and related Fractional Inequalities, TJMM5 (2013), 85-89.

[5] Belarbi, S. and Dahmani, Z., On some new fractional integral inequalities, J. Ineq. Pure Appl. Math. 10 (3) (2009), Art. 86.

[6] H. Budak and P. Agarwal, New generalized midpoint type inequalities for fractional integral, Miskolc Mathematical Notes, 20(2), 2019, 781-793.

[7] F. Chen, A note on the Hermite-Hadamard inequality for convex functions on the co-ordinates, J. Math. Inequal., 8(4), (2014), 915-923.

[8] S. S. Dragomir, On Hadamard's inequality for convex functions on the co-ordinates in a rectangle from the plane, Taiwanese $\mathbf{J}$ Math., 4 (2001), 775-788.

[9] S.S. Dragomir and r. P. Agarwal, Two inequalities for differentiable mappings and applications to special means of real numbers and to trapezoidal formula, Appl. Math. lett. 11 (5) (1998) 91-95.

[10] r. Gorenflo and F. Mainardi, Fractional calculus: integral and differential equations of fractional order, Wien: Springer-Verlag, (1997), 223-276.

[11] M. Iqbal, S. Qaisar and M. Muddassar, A short note on integral inequality of type Hermite-Hadamard through convexity, J. Computational analaysis and applications, 21(5), 2016, pp.946-953.

[12] A. A. Kilbas, H. M. Srivastava and J. J. Trujillo, Theory and applications of fractional differential equations, North-Holland Mathematics Studies, 204, Elsevier Sci. B.V., Amsterdam, 2006.

[13] U. S. Kirmaci, Inequalities for differentiable mappings and applications to special means of real numbers to midpoint formula, Appl. Math. Comput., vol. 147, no. 5, pp. 137-146, 2004, doi: 10.1016/S0096-3003(02)00657-4.

[14] M. A. Latif and S. S. Dragomir, On some new inequalities for differentiable co-ordinated convex functions, Journal of Inequalities and Applications, 2012(1), 2012, 1-13

[15] M. V. Marcela, New inequalities for co-ordinated convex functions via riemann-Liouville fractional calculus, Tamkang Journal of Mathematics, 45(3), 2014, 285-296.

[16] S. Miller, B. ross, An introduction to the fractional calculus and fractional differential equations, New York:Wiley, 1993.

[17] M. E. Ozdemir, C. Yildiz and A. O. Akdemir, On some new Hadamard-type inequalities for co-ordinated quasi-convex functions, Hacettepe Journal of Mathematics and Statistics, 41(5), 2012, 697-707.

[18] Gorenflo, r. and Mainardi, F., Fractional Calculus: Integral and Differential Equations of Fractional Order, Springer Verlag, Wien, 1997, 223-276.

[19] P. L. Butzer, A. A. Kilbas and J.J. Trujillo, Compositions of Hadamard-type fractional integration operators and the semigroup property, Journal of Mathematical Analysis and Applications, 269, (2002), 387-400.

[20] P. L. Butzer, A. A. Kilbas and J.J. Trujillo, Fractional calculus in the Mellin setting and Hadamard-type fractional integrals, Journal of Mathematical Analysis and Applications, 269, (2002), 1-27.

[21] P. L. Butzer, A. A. Kilbas and J.J. Trujillo, Melling transform analysis and integration by parts for Hadamard-type fractional integrals, Journal of Mathematical Analysis and Applications, 270, (2002), 1-15.

[22] U.N. Katugampola, New Approach to a generalized fractional integral, Appl. Math. Comput. 218(3), (2011), 860-865.

[23] K. B. Oldham and J. Spanier, The fractional calculus, Acamedic Press, New York,1974.

[24] S.G. Samko, A.A. Kilbas and O.I. Marichev, Fractional Integrals and Derivatives, Theory and Applications, Gordon and Breach, Yverdon et alibi, 1993.

[25] M.Z. Sarıkaya and H. Ogunmez, On new inequalities via riemann-Liouville Fractional Integration, arXiv:1005.1167v1 [math.CA] 7 May 2010.

[26] V.Kiryakov, Generalized fractional calculus and applications, John Wiley and Sons Inc., New York,1994.

[27] A. A. Kilbas, H.M. Srivastava and J.J. Trujillo, Theory and Applications of Fractional Diferential Equations, Elsevier B.V., Amsterdam, Netherlands, 2006 
[28] M. Z. Sarikaya, Ostrowski type inequalities involving the right Caputo fractional derivatives belong to L_ $\{p\}$, Facta Universitatis, Series Mathematics and Informatics, Vol. 27 No 2 (2012), 191-197.

[29] M. Z. Sarikaya and H. Yaldiz, On weighted Montogomery identities for riemann-Liouville fractional integrals, Konuralp Journal of Mathematics, Volume 1 No. 1 pp. 48-53, 2013.

[30] M. Z. Sarikaya and H. Yaldiz, New generalization fractional inequalities of Ostrowski-Grüss type, Lobachevskii Journal of Math., 2013, Vol. 34, No. 4, pp. 326-331.

[31] M. Z. Sarikaya and H. Filiz, Note on the Ostrowski type inequalities for fractional integrals, Vietnam Journal of Mathematics, January 2014, DOI 10.1007/s10013-014-0056-4.

[32] S. Qaisar and S. Hussain, On hermite-hadamard type inequalities for functions whose first derivative absolute values are convex and concave, Fasciculi Mathematici, 58(1), 2017, 155-166.

[33] M. Z. Sarikaya, E. Set, H. Yaldiz, N. Basak, Hermite-Hadamard's inequalities for fractional integrals and related fractional inequalities, Math Comput Model, 57 (9-10) (2013), 2403-2407.

[34] M. Z. Sarikaya, E. Set, M. E. Özdemir and S. S. Dragomir, New some Hadamard's type inequalities for co-ordinated convex functions, Tamsui Oxf. J. Inf Math Sci. 2012, 28(2), 137-152.

[35] M. Z. Sarıkaya, On the Hermite-Hadamard-type inequalities for co-ordinated convex function via fractional integrals, Integral Transforms and Special Functions, 25(2), 2014, 134-147.

[36] T. Tunç, M. Z. Sarikaya and H. Yaldiz, Fractional Hermite-Hadamard's type inequality for co-ordinated convex functions, TWMS J. Pure Appl. Math., 11(1), 2020, 3-29.

[37] M.A. Latif and S.S. Dragomir, New ostrowski type inequalities for co-ordinated s-convex functions in the second sense, le matematiche, Vol 67 No 1 (2012). 